\title{
Performance Boost of Block Truncation Coding based Image Classification using Bit Plane Slicing
}

\author{
H. B. Kekre, PhD. \\ Senior Professor \\ Dept. of Computer \\ Engineering, \\ MPSTME, \\ SVKM'S NMIMS \\ (Deemed to be \\ University), \\ Mumbai
}

\author{
Sudeep Thepade, \\ PhD \\ Professor \\ Dept. of Computer \\ Engineering, Pimpri \\ Chinchwad College Of \\ Engineering, Pune
}

\author{
Rik Kamal Kumar \\ Das \\ Research Scholar, \\ A.K. Choudhury School of \\ Information Technology, \\ University of Calcutta \\ Assistant Professor \\ Dept. of Information \\ Technology, MPSTME, \\ SVKM's NMIMS (Deemed \\ to be University), \\ Mumbai
}

\author{
Saurav Ghosh \\ Assistant Professor \\ A.K. Choudhury School of \\ Information Technology, \\ University of Calcutta, \\ Kolkata
}

\begin{abstract}
Image classification demands major attention with increasing volume of available image data. The paper has shown performance boosting of image classification after associating Bit Plane Slicing with Block Truncation Coding (BTC) for feature extraction. Here more significant bit planes were considered for extraction of feature vectors. RGB color space was considered to carry out the experimentation. A database of 900 images was used for evaluation purpose.
\end{abstract}

\section{Keywords}

Bit Plane Slicing, BTC, CBIC, RGB

\section{INTRODUCTION}

Information associated to image has got diversified applications like entertainment, education, military services, criminology etc. Image databases are maintained to store these images in digital form. Huge amount of image data is generated everyday due to rapid evolution of image capturing devices.[1] Classification of images inside the database stimulates the searching process efficiency. Image classification creates an organized database by cataloging the images into analogous categories and objects. An image database with classified categories can have limited major classes to search from and it restricts the search only to the class of interest. Image Classification improves the speed of retrieval with better user satisfaction possibility.[2]

\section{IMAGE CLASSIFICATION}

Image classification requires extraction of feature vector from a heterogeneous collection of images. Color, shape and texture of an image are considered as the features used to classify images into limited categories. Averaging and Histogram techniques are used to derive the color feature of an image.[3,4,5] Texture is computed by using vector quantization and gradient and morphological operator is used for formulating the shape feature. $[\mathbf{6 , 7 , 8 , 9 , 1 0 ]}$ Earlier approaches have implemented image classification with $\mathrm{K}$ means clustering with Block Truncation Coding and color moments.[11] Block Truncation Coding (BTC) has been used for image classification using six assorted color spaces alias RGB, Kekre's LUV color space, YCbCr, YUV, YIQ, Kekre's $\mathrm{YCgCb}$ solor space.[12]

\subsection{Block Truncation Coding}

Block Truncation coding is a simple compression algorithm which segments the image into nxn (typically $4 \times 4$ ) non overlapping blocks. [13, 14] In the year 1979 the algorithm was developed at the early stage of image processing. It was developed for the grayscale images and later extended for color images. In this algorithm the blocks are coded one at a time. The reconstructed block comprises of new values calculated from the mean and standard deviation for each block. The value of mean and standard deviation remains same as of the original block. 


\subsection{Bit Plane Slicing}

Bit Plane Slicing considers an image is segmented into different planes starting from Least Significant Bit (LSB) to Most Significant Bit (MSB).

An 8 bit binary vector can be used to denote the intensity value of each pixel and the value of each bit is either 0 or 1 . Each bit plane can be represented as a binary matrix. $[\mathbf{1 5 , 1 6 ]}$. This binary matrix is used further to generate image slices for the respective bit planes. The figure 1 shows the original image along with image slices for higher bit planes, also the image slice generated by accumulation of higher bit planes is shown.

The bit plane of the image can be given as equation 1 where the original image is given as $\mathrm{I}(\mathrm{m}, \mathrm{n}), \mathrm{R}$ is given as Remainder and floor(i) stands for round the elements to I nearest integers less than or equal to $i$.

The equation of bit plane is given in equation 1

$$
\operatorname{Ibp}(m, n)=R\left\{\frac{1}{2} \operatorname{floor}\left[\frac{1}{2^{m}} I(m, n)\right]\right\}
$$

For each color component ( in the considered R,G, B colorspace) there are 8 bit planes. So for the image 24 bit planes are possible, here the higher bit planes are considered to generate image slices which are finally clubbed together for feature extraction as shown in Fig. 1

\section{Fig 1}

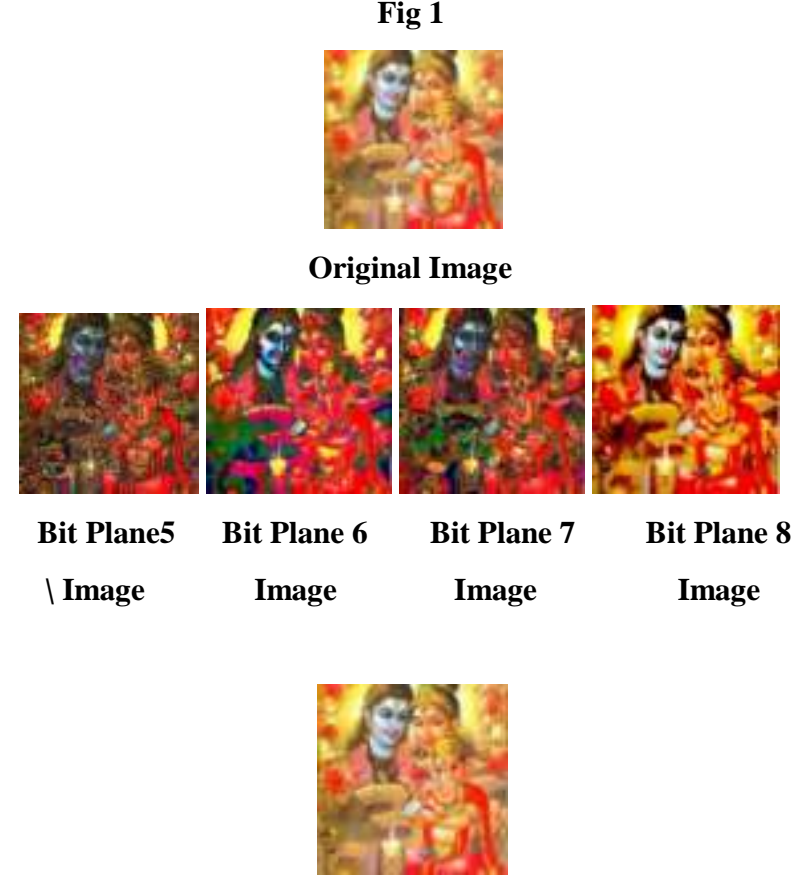

Amalgamated Image of Bit Plane 5,6,7 and 8

\section{PROPOSED \\ IMAGE CLASSIFICATION TECHNIQUE}

RGB color space is considered in this work which consists of the base colors, viz., $\operatorname{red}(\mathrm{R})$, green $(\mathrm{G})$ and blue(B). Mixture of these base colors in full intensities produces white color and if none is used it gives black color. Any desired color can be created if these base colors are mixed in different intensities. [17]. Average intensity value of each image is calculated for each of the R, G and B color planes for all the images in the database[18]. The formulae are given in equations 2,3 and 4 .

$$
\begin{aligned}
& \operatorname{Tav} R=(1 /(m * n)) * \sum_{i=1}^{m} \sum_{j=1}^{n} R(i, j) \\
& \operatorname{Tav} G=(1 /(m * n)) * \sum_{i=1}^{m} \sum_{j=1}^{n} G(i, j) \\
& \operatorname{Tav} B=(1 /(m * n)) * \sum_{i=1}^{m} \sum_{j=1}^{n} B(i, j)
\end{aligned}
$$

Each pixel value is compared with the derived mean value (TavR or TavG or TavB) to divide the image into two sections of upper intensity and lower intensity values respectively. Equations 5, 6 and 7 are used to derive the upper and lower binary planes $(' 1$ '=higher than mean value, ' 0 '=lower than mean value) for respective color components.

$$
\begin{aligned}
& 1, \text { if } \ldots . . R(i, j)>=\operatorname{Tav} R \\
& \operatorname{BBr}(i, j)=\{ \\
& 0, \ldots . . \text { if } \ldots R(i, j)<\operatorname{Tav} R \\
& 1, \text { if } \ldots . . R(i, j)>=\operatorname{Tav} G \\
& B B g(i, j)=\{ \\
& 0, \ldots . i f \ldots R(i, j)<\operatorname{Tav} G \\
& 1, \text { if } \ldots . . R(i, j)>=\operatorname{Tav} B \\
& B B b(i, j)=\{
\end{aligned}
$$


The mean upper and mean lower intensity values for the respective color planes are calculated as per the equation 8 to equation 13 .

$$
\begin{aligned}
& \text { Rlomean }=\frac{1}{m * n-\sum_{i=1}^{m} \sum_{j=1}^{n} B B r(i, j)} * \sum_{i=1}^{n} \sum_{j=1}^{m}(1-B B r(i, j)) * R(i, j) \\
& \text { Glomean }=\frac{1}{m^{*} n-\sum_{i=1}^{m} \sum_{j=1}^{n} B B g(i, j)} * \sum_{i=1}^{n} \sum_{j=1}^{m}(1-B B g(i, j)) * G(i, j) \\
& \text { Blomean }=\frac{1}{m * n-\sum_{i=1}^{m} \sum_{j=1}^{n} B B b(i, j)} * \sum_{i=1}^{n} \sum_{j=1}^{m}(1-B B b(i, j)) * B(i, j) \\
& \text { Bupmean }=\left(\frac{1}{\sum_{i=1}^{m} \sum_{j=1}^{n} B B b(i, j)}\right) * \sum_{i=1}^{m} \sum_{j=1}^{n} B B b(i, j) * B(i, j,
\end{aligned}
$$

\section{Each color component of individual image is sliced into 8}

bit planes. Value of the higher four bit planes viz., plane numbers 5, 6, 7 and 8 are checked for each pixel of each color plane. The mean value of those pixels are calculated whose any of the values of bit plane 5 or bit plane 6 or bit plane 7 or bit plane 8 are equal to 1 as shown by equations $14,15,16$

Comparison of each pixel is done with the mean pixel value thus obtained and two groups of pixels having higher value

$$
B P a v R_{b p 5=1 o r b p 6=1 o r b p 7=1 o r b p 8=1}=\left(1 / m^{*} n\right) * \sum_{i=1}^{m} \sum_{j=1}^{n} R(i, j)
$$

$$
B P a v G_{b p 5=1 o r b p 6=10 r b p 7=1 o r b p 8=1}=(1 / m * n) * \sum_{i=1}^{m} \sum_{j=1}^{n} G(i, j)
$$

$$
B P a v B_{b p 5=1 \text { orbp } 6=1 \text { orbp } 7=1 \text { orbp } 8=1}=\left(1 / m^{*} n\right) * \sum_{i=1}^{m} \sum_{j=1}^{n} B(i, j)
$$

than the mean pixel and lower value than the mean pixel are derived. The equations are given in equation 17,18 and 19

$$
1, \text { if } \ldots . . R(i, j)>=B P a v R
$$

$\operatorname{BPBr}(i, j)=\{$

$$
0, \ldots . i f \ldots R(i, j)<B P a v R
$$

$$
1, \text { if } \ldots . . R(i, j)>=\text { BPavG }
$$

$$
\operatorname{BPBg}(i, j)=\{
$$

$$
0, \ldots \text { if } \ldots R(i, j)<B P a v G
$$

$$
1, \text { if } \ldots . R(i, j)>=B P a v B
$$

$B P B b(i, j)=\{$

$$
0, \ldots . \text { if } \ldots R(i, j)<B P a v B
$$

Mean upper pixel value and mean lower pixel value is calculated from the pixels having higher values than mean value and from the pixels having lower values than mean value respectively, as given in equations 20 to 25 . 


$$
\text { Rbpupmean }=\frac{1}{\sum_{i=1}^{m} \sum_{j=1}^{n} B \operatorname{PBr}(i, j)} * \sum_{i=1}^{m} \sum_{j=1}^{n} B \operatorname{PBr}(i, j) * R(i, j)
$$

Gbpupmean $=\frac{1}{\sum_{i=1}^{m} \sum_{j=1}^{n} B P B g(i, j)} * \sum_{i=1}^{m} \sum_{j=1}^{n} B P B g(i, j) * G(i, j)$

Bbpupmean $=\left(\frac{1}{\sum_{i=1}^{m} \sum_{j=1}^{n} B P B b(i, j)}\right) * \sum_{i=1}^{m} \sum_{j=1}^{n} B P B b(i, j) * B(i, j)$

$$
\text { Rbplomean }=\frac{1}{m * n-\sum_{i=1}^{m} \sum_{j=1}^{n} B P B r(i, j)} * \sum_{i=1}^{n} \sum_{j=1}^{m}(1-\operatorname{BPBr}(i, j)) * R(i, j)
$$$$
\text { Gbplomean }=\frac{1}{m * n-\sum_{i=1}^{m} \sum_{j=1}^{n} \operatorname{BPBg}(i, j)} * \sum_{i=1}^{n} \sum_{j=1}^{m}(1-\operatorname{BPBg}(i, j)) * G(i, j)
$$$$
\text { Bbplomean }=\frac{1}{m * n-\sum_{i=1}^{m} \sum_{j=1}^{n} B P B b(i, j)} * \sum_{i=1}^{n} \sum_{j=1}^{m}(1-B P B b(i, j)) * B(i, j)
$$

The four mean color values thus derived forms the feature vector of the image. These feature vectors are calculated for all the images in the database.

\section{RESULTS IMPLEMENTATION}

AND

Image Classification using BTC with Bit plane slicing is implemented with Intel core 2 duo processor with 1 GB RAM. The experiment is carried out in Matlab 7.11.0(R2010b). The proposed classification method is tested on an image database of 900 images. The image database has nine different categories. Each category comprises of hundred images. Some categories of the image database are taken from Wang's database[19]. The nine different categories are Ganeshii, Sea Beaches, Sunflower, Candles, Dinosaurs, Elephants, Roses, Horses and Mountains.

The classification success rate is found out by firing 900 queries on the image database. Performance comparison of the proposed image classification method is done by considering the average classification rate of all these queries.
SuRate $=\frac{\text { No. of queries classified }}{\text { Total no. of queries considered for classification }}$

The sample ensemble of images from the considered image database used in the database is given in Fig 2

Fig .2

\section{Sample Image Database}

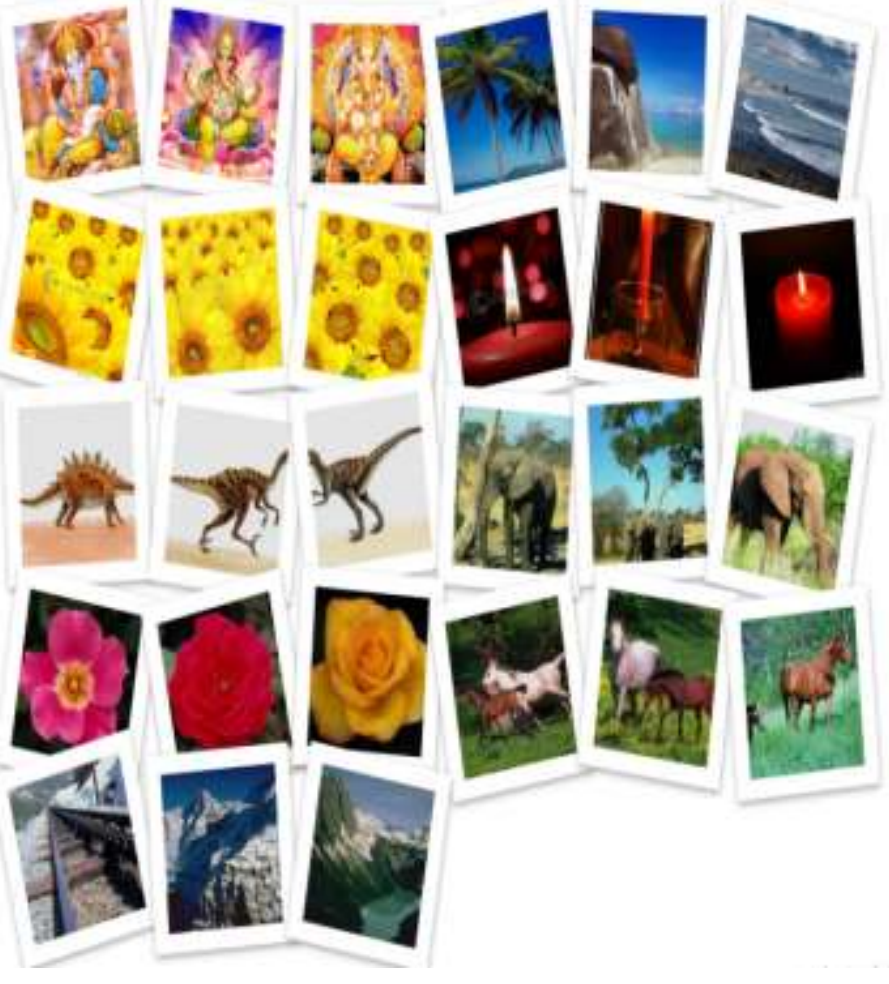

\subsection{Performance Measure}

Mean Square Error (MSE) method is followed to compare the feature vectors of the database images with the query images. Equation 26 gives the formula for calculating MSE.

$$
M S E=\frac{1}{M N} \sum_{y=1}^{M} \sum_{x=1}^{N}\left[I(x, y)-I^{\prime}(x, y)\right]^{2}
$$

Performance measure of the technique is denoted by the success rate for the queries classified. The success rate is shown in equation 27 
Comparison of average success rate is shown according to image categories in Table 1 for btc based image classification without bit plane slicing and with bit plane slicing

Table 1

Comparison of Average Success Rate

\begin{tabular}{|l|c|c|}
\hline \multirow{2}{*}{ Categories } & \multicolumn{2}{|c|}{ Success Rate } \\
\cline { 2 - 3 } & $\begin{array}{c}\text { BTC without } \\
\text { Bit Plane } \\
\text { Slicing }\end{array}$ & $\begin{array}{c}\text { BTC with Bit } \\
\text { Plane Slicing }\end{array}$ \\
\hline 1(Ganeshji) & 82 & 86 \\
\hline 2 (Sea Beach) & 61 & 62 \\
\hline 3 (Sunflower) & 94 & 97 \\
\hline 4 (Candle) & 69 & 85 \\
\hline 5 (Dinosaur us) & 100 & 100 \\
\hline 6 (Elephant) & 75 & 78 \\
\hline 7 (Roses) & 67 & 76 \\
\hline 8 (Horses) & 88 & 88 \\
\hline 9 (Mountains) & 58 & 50 \\
\hline $\begin{array}{l}\text { Average } \\
\text { Success Rate }\end{array}$ & 77.11 & 80.22 \\
\hline
\end{tabular}

The comparison of percentage success rate is shown in Fig. 3 for proposed image classification using bit plane slicing versus image classification without using bit plane slicing.

Fig. 3

\section{Comparison of Percentage Success Rate of Image Classification}

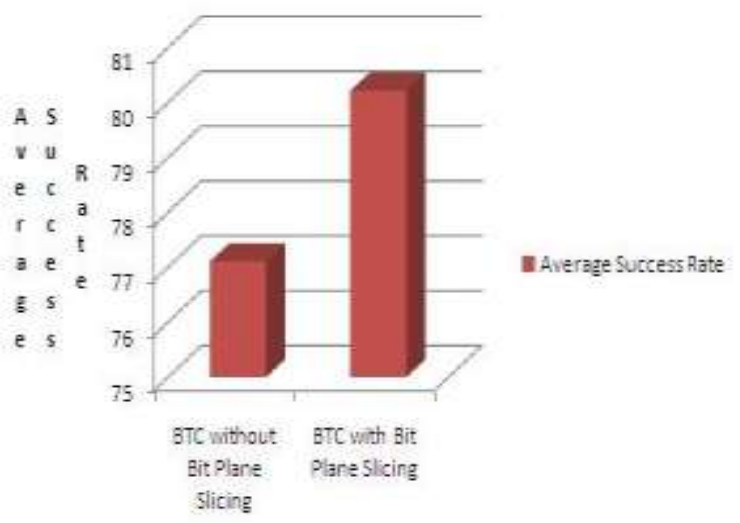

\section{CONCLUSION}

Efficient analysis of image data can provide information of importance. Content based Image classification addresses the problem of efficiency improvement in image retrieval from diverse collection of images in image databases. This paper significantly points out that grouping of images into meaningful classes using BTC with plane slicing based improvises its performance. A database of 900 images is used as test bed for the proposed methodology. The result has shown enhancement in performance compared to the previous method of image classification without using bit plane slicing.

\section{REFERENCES}

[1]. Zakariya, S. M., Ali, R., \& Ahmad, N. . Combining Visual Features of an Image at Different Precision Value of Unsupervised Content Based Image Retrieval. Computational Intelligence and Computing Research (ICCIC)(2011)

[2]. H.B.Kekre, Sudeep D. Thepade, "Image Retrieval using Augmented Block Truncation Coding Techniques", ACM International Conference on Advances in Computing, Communication and Control (ICAC3- 2009), pp. 384-390, 23-24 Jan 2009, Fr. Conceicao Rodrigous College of Engg., Mumbai. Is uploaded on online ACM portal.

[3]. H.B.kekre, Sudeep D. Thepade, "Improving Color to Gray and Back using Kekre's LUV Color Space", IEEE International Advanced Computing Conference 2009 (IACC ${ }^{\text {ce }} 9$ ), Thapar University, Patiala, INDIA, 6-7 March 2009. Is uploaded and available online at IEEE Xplore.

[4]. Z. Zhang, W.Li \& B. Li, "An Improving Technique of Color Histogram in Segmentation-based Image Retrieval." 2009 Fifth International Conference on Information Assurance and Security,381-384(2009)

[5]. R. Chakravarti, Meng Xiannong, "A Study of Color Histogram Based Image Retrieval," 2009 Sixth International Conference on Information Technology: New Generations, 2009.ITNG '09., 1323-1328(2009)

[6]. Dr.H.B.Kekre, Sudeep D. Thepade, "Image Retrieval using Non-Involutional Orthogonal Kekre"s Transform", International Journal of Multidisciplinary Research and Advances in Engineering (IJMRAE), Ascent Publication House, 2009, Volume 1, No.I, pp 189-203, 2009. Abstract available online at www.ascent-journals.com

[7]. Maheshwari, M., Silakari, S., and Motwani, M. (2009). Image Clustering Using Color and Texture. 2009 First International Conference on Computational Intelligence Communication Systems and Networks,403-408.

[8]. Dr.H.B.Kekre, Sudeep D. Thepade, "Image Retrieval using Color-Texture Features Extracted from Walshlet Pyramid", ICGST International Journal on Graphics, Vision and Image Processing (GVIP), Volume 10, Issue I, Feb.2010, pp.9-18, 
www.icgst.com/gvip/Volume10/Issue1/P1150938876.

html

[9]. Dr.H.B.Kekre, Tanuja K. Sarode, Sudeep D. Thepade, Vaishali Suryavanshi,"Improved Texture Feature Based Image Retrieval using Kekre"s Fast Codebook Generation Algorithm", Springer-International Conference on Contours of Computing Technology (Thinkquest-2010), Babasaheb Gawde Institute of Technology, Mumbai, 13-14 March 2010, The paper will be uploaded on online Springerlink.

[10]. Wu Yanyan, Wu YIquan, "Shape-Based Image Retrieval Using Combining Global and Local Shape Features" 2nd International Congress on Image and Signal Processing, 2009. CISP '09. pp 1-5, 2009

[11]. Dr.H.B.Kekre, Sudeep D. Thepade, "Boosting Block Truncation Coding using Kekre"s LUV Color Space for Image Retrieval", WASET Int. Journal of Electrical, Computer and System Engineering (IJECSE), Volume 2, Number 3, pp. 172-180, Summer 2008.

[12]. Dr. H.B. Kekre. Dr. Sudeep D. Thepade, Rik Kamal Kumar Das, Saurav Ghosh, " Image Classification using Block Truncation Coding with Assorted Color Space", International Journal of Computer Applications(IJCA), April 2012 edition

[13] H.B.Kekre, Sudeep D. Thepade, Varun Lodha, Pooja Luthra, Ajoy Joseph, Chitrangada Nemani "Augmentation of Block Truncation Coding based
Image Retrieval by using Even and Odd Images with Sundry Colour Space" Int. Journal on Computer Science and Engg. Vol. 02, No. 08, 2010, 2535-2544

[14] H.B.Kekre, Sudeep D. Thepade, Shrikant P. Sanas Improved CBIR using Multileveled Block Truncation Coding International Journal on Computer Science and Engineering Vol. 02, No. 08, 2010, 2535-2544

[15] Rafael C. Gonzalez and Richard Eugene Woods "Digital Image Processing", 3rd edition, Prentice Hall, Upper Saddle River, NJ, 2008. ISBN 0-13-168728-X. pp. 407-413.S

[16] S.Sridhar " Digital Image Processing", $1^{\text {st }}$ edition, Oxford University Press, YMCA Library Building, Jai Singh Road, New Delhi 110001, ISBN 0-19-807078-0 , pp. 188,237

[17] Mark D. Fairchild, "Colour Appearance Models", 2nd Edition, Wiley-IS\&T, Chichester, UK, 2005. ISBN 0470-01216-1

[18]. Dr.H.B.Kekre, Sudeep D. Thepade, "Using YUV Color Space to Hoist the Performance of Block Truncation Coding for Image Retrieval", IEEE International Advanced Computing Conference 2009 (IACC ${ }^{\text {ee }}$ 09), Thapar University, Patiala, INDIA, 6-7 March 2009.

[19]. Image Database http://wang.ist.psu.edu/docs/related/Image.orig(last referred on 20/05/2012) 